\title{
The increase in urinary serotonin and decrease in salivary cortisol concentrations following direct inhalations of concentrated essential oils is not induced by non-specific effects
}

\author{
Rainer Schneider \\ RECON - Research and Consulting, Teningen, Germany \\ E-mail: info@recon-freiburg.biz
}

Objectives. The effectiveness of exogenously triggered serotonin (e.g., dietary supplements, drugs) increase is varied. However, since urinary serotonin concentrations were found to correlate with those in the cerebrospinal fluid, the olfactory system might be an efficient and testable pathway to quickly elevate serotonin levels due to its fast-acting central neurophysiological and peripheral pathways. However, little research has been devoted to investigate this assumption. This paper extends previous findings of parasympathetic activation of a specially designed essential oil inhaler (AromaStick ${ }^{\odot}$ Balance) by experimentally testing its impact on urine serotonin and saliva cortisol excretion.

Method. Two experiments involving healthy individuals were conducted to test the efficacy of essential oil application to the nose by employing different inhalation protocols and control conditions.

Results. In the pilot study $(\mathrm{n}=8)$, serotonin urine excretion was increased after six inhalations (effect size Cohen's $d=0.7)$. In the second experiment $(n=80)$, inhalations proved superior to both the natural control condition and the pseudo placebo condition after three and six inhalation cycles $(0.6<\mathrm{d}<1.8)$. In addition, there was a large reduction of cortisol saliva levels after three inhalations $(\mathrm{d}=0.9)$.

Conclusion. Short and deep inhalations of essential oil scents directly delivered to the olfactory system appear to result in an enhanced serotonin and a reduced cortisol release in healthy individuals of both sexes.

Key words: AromaStick ${ }^{\circ}$, cortisol, essential oil inhaler, placebo effects, serotonin

Serotonin (5-hydroxytryptamine, 5-HT) plays an important role as a neurotransmitter for a wide range of neuropsychological processes and psychological functions (Berger et al. 2009; Del Colle et al. 2020). Popularly referred to as the 'happiness hormone', 5 -HT is best known for its mood-altering property, for instance, by counteracting aversive emotional states (Strasser et al. 2016). To treat a deficiency in serotonin, dietary recommendations focus on foods rich in tryptophan, which, unlike serotonin, crosses the blood-brain barrier. Foods rich in carbohydrates can increase the release of serotonin and may have similar mood-enhancing effects (e.g., serenity, inner peace and contentment). However, the effectiveness of dietary changes and amino acid supplementation is varied and subject to a number of moderating and modulating factors, among which are tolerance and interindividual differences in responsiveness. Additionally, high-dose or long-term supplementation of 5-hydroxytryptophan (5-HTP) over one month may cause significant side effects and adverse reactions, e.g., due to the depletion of catecholamines 
(Hinz et al. 2012). Although there is consensus that amino acid precursors elevate serotonin levels within the critical brain tissues and organs, their mode of action in the body is not fully understood. A study involving more than 800 individuals ingesting 5-HTP on a daily basis found inverse, direct, and no relationships between urinary serotonin excretion and 5-HTP doses (Trachte et al. 2009). Consumption of serotonin precursors only increased urinary excretion approximately $50 \%$ of the time, and up to $40 \%$ of the participants responded with no response or a reduction. These results could be somewhat due to part of serotonin in the kidneys being catabolized or reabsorbed into the bloodstream instead of being effluxed in the urine.

In the clinical realm, synthetic antidepressant drugs like monoamine oxidase inhibitors, tricyclic antidepressants, and selective serotonin reuptake inhibitors are the most widely prescribed for increasing $5-\mathrm{HT}$ concentration in the brain. Yet, their effectiveness also varies and when used long-term can result in severe side effects like fatigue, headache, constipation, overweight, and tachycardia (Santarsieri and Schwartz 2015). In a large meta-analysis involving more than 6,000 children and adolescents, the effect size for the specific treatment of depressive disorders was only small compared to placebo treatment whilst the effect sizes for treatment-induced severe adverse events was very large (Locher et al. 2017).

Among alternative medical approaches, psychostimulant essential oils may constitute a safe and natural alternative to stimulate 5-HT release. Research in clinical aromatherapy indicates that some essential oils help to reduce anxiety, depression, and stress (Agatonovic-Kustrin et al. 2020; Edris 2007; Lv et al. 2013). The olfactory tract, located in the olfactory sulcus of the basal forebrain, may particularly be suited for a fast-acting modulation of 5-HT production due to direct transmission of information to the orbital prefrontal cortex, the amygdala, the hypothalamus, the basal ganglia, and the hippocampus (Masaoka and Homma 2011), causing odors to act on various neuroendocrine systems (Kiecolt-Glaser et al. 2009). These projections include the raphe nuclei located in the brain stem, which are the major central nervous source of 5-HT (Frazer and Hensler 1999).

Animal research indicates that essential oils such as ylang-ylang produce calming effects by increasing serotonin levels while decreasing glucocorticoids (Zhang et al. 2018). Conversely, acute stress and hypothalamus-pituitary-adrenal (HPA) axis activation associated with increased production of cortisol enhances the synthesis of the serotonin transporter (SERT), which decreases serotonin concentration in the synaptic cleft (Tafet et al. 2001). Inhalation of essential oils not only affects the brain, but also a variety of body systems. For instance, odor molecules are detectable in the blood circulation shortly after inhalation, altering blood composition (Nakumara et al. 2009; Schneider 2017). Moreover, olfactory cells are found on the surface of many organs including the gastrointestinal tract. Approximately $90 \%$ of total serotonin quantity in the human body is located in the enterochromaffin cells in the gastrointestinal tract, and essential oil molecules stimulate serotonin release via this pathway. $\mathrm{Ca}^{2+}$ imaging studies have shown that odorant ligands of the olfactory receptors cause $\mathrm{Ca}^{2+}$ influx, elevation of intracellular free $\mathrm{Ca}^{2+}$ levels, and, consequently, serotonin release (Braun et al. 2007). Enterochromaffin cells are gut chemosensors that couple to bi-directional neural pathways, whereby short intramural and long extramural reflexes are triggered to release various gut hormones, including serotonin (Bellono et al. 2017; Konturek et al. 2004). Serotonin enters the circulation through capillary beds in the submucosa of the intestinal wall. Once in the bloodstream, most serotonin is sequestered by SERT-mediated transport within platelets where vesicular monoamine transporters compartmentalize serotonin into dense granules which allow an efficient transportation throughout the circulation (Yabut et al. 2019). There are, however, many open questions regarding the underlying mechanisms of aromatherapeutic effects on serotonin release in humans. Although meta-analyses and systematic reviews report favorable effects of aromatherapeutic interventions for treating stress (Hur et al. 2014), sleep disorders (Hwang and Shin 2015), or depressive symptoms (Sanchez-Vidana et al. 2017), they do not account for neuroendocrine biomarkers like serotonin.

Recently, several studies involving an essential oil inhaler which delivers short, but highly concentrated scent molecules directly to the nose reported large improvement rates for a variety of parameters (Schneider et al. 2019). In a series of studies exploring parasympathetic effects, a specially formulated inhaler markedly reduced saliva cortisol concentration after ten minutes in individuals with elevated stress levels (Schneider 2016). Although serotonin interacts with cortisol in complex ways, it generally hampers the production of cortisol (Mahar et al. 2014). Thus, it may be conjectured that such an inhaler could have the capability not only to reduce cortisol levels but to increase serotonin levels, as well. 
The aim of this work was to investigate the effects of direct inhalations of essential oil compounds on serotonin and cortisol release. First, a pilot study was conducted to assess the impact of a scent composition with strong parasympathetic effects to increase urinary serotonin levels. Based on the findings of the pilot study, the main study investigated the efficacy of the inhaler in relation to the effect duration after inhalation, comparison to two control conditions and salivary cortisol reduction in non-stressed individuals. In summation, the present work addressed the following questions:

Do the previously demonstrated effects of an essential oil inhaler ('Balance') on neurocardiac mechanisms translate to neuroendocrine mechanisms involving urine serotonin excretion?

If so, to what extent are these effects attributable to non-specific effects (natural fluctuations, placebo effects)?

What is the time dependency of these effects?

\section{Subjects and Methods}

Intervention. Participants applied the inhaler by directly holding it under one nostril with one hand while closing the other nostril with the index finger. The lipstick-sized inhaler contained $100 \%$ natural essential oils from peppermint, cypress, geranium, and ginger (ratio: 48-34-14-4), held in a suspended filter in the center of the tube. The device is fabricated by AromaStick Inc., Sargans, Switzerland (Singer 2016). The essential oils are obtained from ESSENCIA, a Swissmedic approved essential oil supplier. The oils are individually assessed with a Certificate of Analysis (COA) and have an organic certificate issued by bio.inspecta (Switzerland).

\section{Pilot Study}

Participants. A total of 8 participants were enrolled in the pilot study ( 4 females and 4 males). Their mean age was $37.9 \pm 9.6$ years (mean $\pm S D)$. Inclusion in the study was dependent on the following criteria: (a) no disorder of the respiratory system (e.g., asthma, seasonal cold); (b) absence of carcinoid syndrome; (c) no current intake of pharmacologic agents (e.g., antidepressants, dietary tryptophan, ephedrine, melatonin); (d) absence of premenstrual dysphoria, pregnancy, or menses (including one week prior); and (e) no consumption of food and beverages (except water) for 2 hours prior to the experiment. In addition, consumption of foods rich in dietary tryptophan (banana, coffee, chocolate, eggplant, kiwi, nuts, pineapple, plum, tomatoes) was to be abstained for 24 hours prior to the experiment to reduce falsepositive results. All participants provided written informed consent and were remunerated with $20 €$. The study was run following the Ethical Principles for Medical Research Involving Human Subjects of the World Medical Association.

Study design and procedure. All data collections were conducted in a fully ventilated room $\left(30 \mathrm{~m}^{2}\right)$ with constant temperature $\left(20^{\circ} \mathrm{C}\right)$, humidity $(50 \%)$, and room illumination (200 lx), and run between 14:00 p.m. and 17:00 p.m. to account for circadian effects, with the participants sitting upright in a comfortable armchair. The experimenter was blind as to the ingredients of the inhaler.

Ten minutes prior to receiving the intervention, urine samples were collected and acidified with $1 \mathrm{ml} \mathrm{HCL}$ per $100 \mathrm{ml}$ urine (baseline measurement). The intervention consisted of 6 breathing cycles comprising 3 inhalations each of the inhaler "Balance". The first inhalation started 10 minutes after the baseline spot urine collection. After that, additional inhalation cycles followed at minute 20, 30, 40, 50 and 60. Each inhalation consisted of a 3 second inhalation period, a 2 second holding phase and a 2 second exhalation phase through the mouth. Participants alternately inhaled through each nostril. Inhalers were presented without labels and description of their content. Ten minutes after the last inhalation post treatment spot urine collection ensued. Urine samples were collected again and acidified with $1 \mathrm{ml} \mathrm{HCL}$ per $100 \mathrm{ml}$ urine.

Extraction of neuroendocrine biomarker. All blinded samples were analyzed by an independent laboratory (TECAN, Hamburg, Germany) for enzyme immunoassay and in vitro diagnostic and expressed as ng/ml. Sample preparation (derivatization of serotonin to $\mathrm{N}$-acylserotonin) was achieved by incubating the samples with the acylating reagent. In the competitive enzyme immunoassay (ELISA), the biotinylated antigen and the non-biotinylated antigen competed for the limited number of binding sites of the antibody bound to the wells of the microtiter strips. After incubation, the unbound biotinylated antigen was removed by washing. The antibodybound biotinylated antigen was determined by streptavidin alkaline phosphatase as marker and p-nitrophenyl phosphate as substrate.

\section{Main Study}

Participants. Based on the effect size of the pilot study, the sample size of each experimental group was set at $n=20$ to achieve a power of $1-\beta \geq 0.95$ (Faul et al. 2009). In total, 80 participants (40 females and 
40 males) were enrolled in the study 2 . Their mean age was $38.5 \pm 11.4$ years (mean $\pm S D$ ). Inclusion criteria were set to identically reflect those of the pilot study.

Study design and procedure. To ensure comparability of results with the pilot study, the study location, participant setup and storing of the samples was replicated. In addition to the pilot study, participants rated their mood before baseline sampling using a 5-point-Likert Scale with the anchors 'not at all', 'a little', 'moderately', 'pretty much', and 'very much'. The mood items were (1) cheerful, (2) peaceful, (3) joyful, (4) relaxed, (5) calm, (6) anxious, (7) grievous, (8) sad, (9) tense, and (10) displeased. A total score was calculated by recoding items $6,7,8,9$, and 10 and summating all ten items (a higher score indicating better mood). Also, saliva samples were collected for assessing cortisol levels. Participants were randomly assigned to the experimental conditions and parallelized with regard to gender. This was done using a randomized block design containing five-digit random number sequences that were ranked in ascending succession and assigned to the experimental conditions. Before each experimental trial, the experimenter opened an opaque envelope containing the experimental condition the participant was assigned to. Post-treatment mood assessment followed after urine and saliva collection at the end of the session.

Natural control condition. This condition served as the primary control, in which participants $(n=20)$ were not subjected to an intervention. The time lapse between sampling and post-treatment sampling was 70 minutes.

Pseudo-placebo control condition. In the second control condition, participants $(n=20)$ employed an inhaler filled with $0.8 \mathrm{ml}$ espresso blend ('ristretto', Nespresso Inc.) individually prepared 30 minutes prior to the experiment. The inhalation pattern and post-treatment sampling was identical to the pilot study, i.e., 6 inhalation cycles followed baseline sampling at minute 10, 20, 30, 40, 50, and 60. Participants were fully debriefed with regard to the content of the inhaler and the meaning of the intervention. However, the purpose of this condition was to test whether espresso scent had a conditioned or expectancy effect on 5-HT release since caffeine is known to increase serotonin release.

Odor inhaler 'Balance 1'. In this treatment condition, participants used the inhaler 'Balance' in the same fashion as in the pilot study.

Odor inhaler 'Balance 2'. In this treatment condition, participants used the inhaler 'Balance' for only 3 inhalation cycles (i.e., 10 minutes after baseline, and additional inhalation cycles followed at minute 20 and 30).

Extraction of neuroendocrine biomarkers. Serotonin was assessed in accordance with the pilot study. Cortisol levels were assessed using the enzyme immunoassay test (ELISA) following the typical competitive binding scenario between an unlabeled antigen and an enzyme-labeled antigen (conjugate) for a limited number of antibody binding sites on the microplate. After washing and decanting procedures unbound materials were removed. After the washing step, the enzyme substrate was added. The enzymatic reaction was terminated by addition of the stopping solution. The absorbance was measured on a microtiter plate reader and expressed as $\mathrm{ng} / \mathrm{ml}$ (5-HT) and $\mu \mathrm{g} / \mathrm{dl}$.

Data analysis. To assess treatment effects, the effect size $\eta^{2}$ for ANOVAs and the effect size d (Cohen 2008) and confidence intervals (95\%) for betweengroup comparisons were calculated (Borenstein et al. 2009). Generally, an effect of $d=0.2$ is regarded as small, $\mathrm{d}=0.5$ is regarded medium and $\mathrm{d} \geq \mathrm{d}=0.8$ is regarded as large and clinically relevant. Dependent variables were difference scores between baseline and pre-treatment measures. Calculation of effect sizes was in alignment with meta-analytical practice (Hunter and Schmidt 2004), the statistics reported in the review article (Schneider et al. 2019), and as a consequence of the highly problematic use of Null Hypothesis Significance Testing (NHST) (Greenland et al. 2016).

\section{Results}

Pilot Study. Baseline and post-treatment values were normally distributed (K-S $\mathrm{Z}=0.5-0.6)$ and fell within normal boundaries $(28-152 \mathrm{ng} / \mathrm{ml})$. The analysis revealed that repeated inhalations of the odor scents increased urine serotonin excretion within a time window of 70 minutes. On average, serotonin levels increased from baseline to post-treatment sampling from $59.1 \pm 27.8 \mathrm{ng} / \mathrm{ml}($ mean \pm SD) to $95.6 \pm 52.9 \mathrm{ng} / \mathrm{ml}($ mean \pm SD). The increase of $62 \%$ corresponded to an effect of $\mathrm{d}=0.7$ (CI: $0.1<\mathrm{d}<1.3$ ). However, the range of increased serotonin levels varied from $58 \%$ to $198 \%$ indicating strong interindividual differences with regard to responsiveness to essential oil inhalation. Two participants showed a decrease in urine serotonin of $8 \%$ and $49 \%$.

Main Study.

Serotonin. Baseline and post-treatment values were normally distributed (K-S $\mathrm{Z}=0.5-0.6$ ) and 
Table 1

Baseline and post-treatment concentrations of urinary serotonin and saliva cortisol, and mood ratings in different experimental groups

\begin{tabular}{|c|c|c|c|c|c|c|c|c|}
\hline \multirow{2}{*}{ Parameter } & \multicolumn{4}{|c|}{ Baseline $^{\dagger}$} & \multicolumn{4}{|c|}{ Post-Treatment } \\
\hline & Control & Placebo & Balance $1^{\mathrm{a}}$ & Balance $2^{\mathrm{b}}$ & Control & Placebo & Balance 1 & Balance 2 \\
\hline Serotonin $(\mathrm{ng} / \mathrm{ml})$ & $80.7 \pm 35.4$ & $77.3 \pm 22.9$ & $66.9 \pm 25.7$ & $73.9 \pm 28.5$ & $76.2 \pm 33.9$ & $69.3 \pm 20.5$ & $85.9 \pm 28.2$ & $86.8 \pm 37.1$ \\
\hline Cortisol $(\mu \mathrm{g} / \mathrm{dl})$ & $0.17 \pm 0.15$ & $0.16 \pm 0.13$ & $0.23 \pm 0.22$ & $0.28 \pm 0.36$ & $0.16 \pm 0.15$ & $0.16 \pm 0.13$ & $0.14 \pm 0.14$ & $0.10 \pm 0.13$ \\
\hline Mood (Range scale: $10-50$ ) & $29.1 \pm 6.4$ & $30.3 \pm 7.5$ & $33.8 \pm 7.8$ & $32.9 \pm 8.1$ & $30.6 \pm 4.3$ & $32.5 \pm 5.2$ & $40.1 \pm 5.5$ & $38 \pm 5.9$ \\
\hline
\end{tabular}

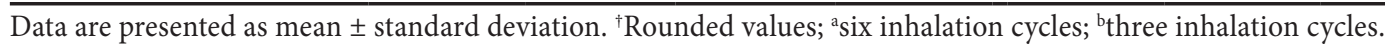

fell with normal boundaries (26-171 ng/ml). As depicted in Table 1 and Figure 1, the mean 5-HT level in the natural control condition decreased from $80.7 \mathrm{ng} / \mathrm{ml}$ in the baseline sampling to 76.2 $\mathrm{ng} / \mathrm{ml}$ in the pre-treatment sampling. Likewise, the serotonin urine concentration in the placebo group dropped from $77.3 \mathrm{ng} / \mathrm{ml}$ to $69.3 \mathrm{ng} / \mathrm{ml}$. In contrast, the 5-HT concentration in both inhaler conditions increased considerably from $66.9 \mathrm{ng} / \mathrm{ml}$ to $85.9 \mathrm{ng} /$ $\mathrm{ml}$ (Balance 1; six inhalation cycles), and from 73.9 $\mathrm{ng} / \mathrm{ml}$ to $86.8 \mathrm{ng} / \mathrm{ml}$ (Balance 2; three inhalation cycles). The four conditions did not differ at baseline $\left(\mathrm{F}[3,76]=0.9 ; \eta^{2}=0.03\right)$, but the ANOVA for the difference scores (post-treatment minus baseline) revealed a large effect of $\eta^{2}=0.16 \quad[F(3,56)=4.8]$. Single mean difference comparisons showed that the Balance 1 group largely differed from the natural control group ( $\mathrm{d}=0.8$; CI: $0.2<\mathrm{d}<1.5)$ and the placebo group $(\mathrm{d}=1.1 ; \mathrm{CI}: 0.5<\mathrm{d}<1.8)$. The difference between the Balance 2 group and the natural control group was medium in size $(\mathrm{d}=0.6$; CI: $0<\mathrm{d}<1.2)$ and the difference between the Balance 2 group and the placebo group was large $(\mathrm{d}=0.9$ : CI: $0.2<\mathrm{d}<1.9)$. The natural control group and the placebo group did not differ $(\mathrm{d}=0.1 ; \mathrm{CI}:-0.5<\mathrm{d}<0.7)$ indicating that sniffing the espresso blend did not produce any placebo effects. With regard to relative urine serotonin release, six inhalation cycles increased 5-HT by approx. 28\% while three inhalation cycles increased it by about $18 \%$. Three individuals in the Balance 1 condition showed a decrease of 5 -HT between $4 \%$ and $50 \%$, the rest of the sample had an increase from 5\% to $154 \%$. In the Balance 2 condition, five participants showed a decrease of $12 \%$ to $86 \%$, and the remaining individuals showed an increase of $11 \%$ to $206 \%$.

Cortisol. All data fell within normal boundaries $(0.02-1.1 \mu \mathrm{g} / \mathrm{dl})$ but both the baseline and the posttreatment values failed to be normally distributed (K-S $Z=1.8-2.4)$. Due to that fact both variables were log-transformed before they were subjected to further analyses. The ANOVA for the difference scores (posttreatment minus baseline) revealed a medium to large effect of $\eta^{2}=0.09 \quad[F(3,56)=2.6]$. Single mean comparisons showed that the natural control group and the placebo group had almost identically slight reductions in cortisol levels ( $\mathrm{d}=0.01$; $\mathrm{CI}:-0.6<\mathrm{d}<0.6)$. In contrast, the reduction in both inhaler groups was

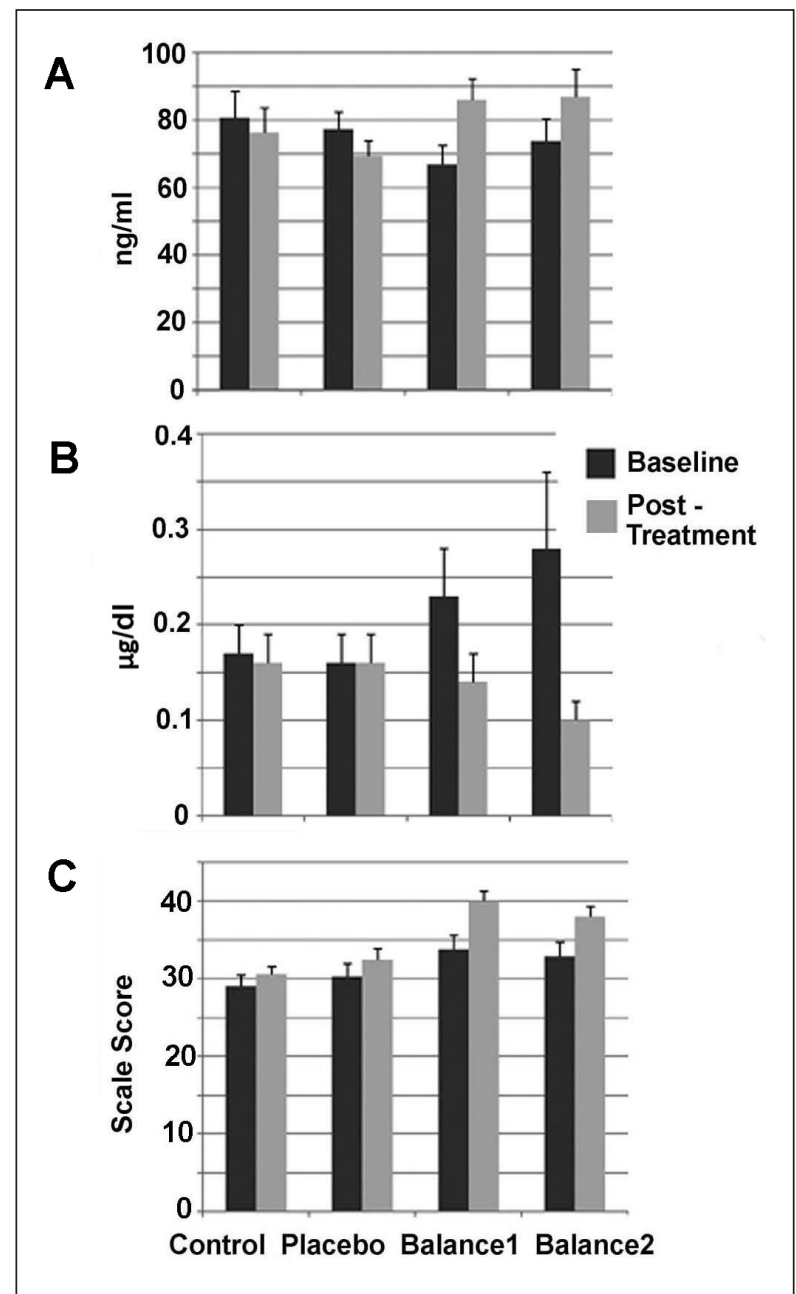

Figure 1. Baseline and post-treatment values of urinary serotonin $(\mathbf{A})$, saliva cortisol $(\mathbf{B})$ and mood ratings $(\mathbf{C})$ in participants under different experimental conditions in the main study. Data are presented as mean and standard errors $(n=80)$. 
markedly larger (Table 1). The mean cortisol level in the Balance 1 condition decreased from $0.23 \mu \mathrm{g} / \mathrm{dl}$ in the baseline sampling to $0.14 \mu \mathrm{g} / \mathrm{dl} \mathrm{l}$ in the pre-treatment sampling and in the Balance 2 condition form $0.28 \mu \mathrm{g} / \mathrm{dl}$ to $0.1 \mu \mathrm{g} / \mathrm{dl}$. However, only the Balance 2 condition (three inhalation cycles) differed from the natural control condition $(\mathrm{d}=0.7$; CI: $0.1<\mathrm{d}<1.3)$ and the placebo condition $(\mathrm{d}=0.9$; CI: $0.3<\mathrm{d}<1.6)$. The differential effects for the Balance 1 group and both control groups were medium in size and fell within positive and negative confidence intervals (Balance 1 - Placebo: $\mathrm{d}=0.6$; CI: $-0.1<\mathrm{d}<1.2$; Balance 1 - Natural Control: $\mathrm{d}=0.5$; CI: $-0.1<\mathrm{d}<1.1)$. However, the difference between the Balance 1 and 2 groups was negligible $(\mathrm{d}=0.2$; CI: $-0.4<\mathrm{d}<0.9)$.

Mood. Baseline and post-treatment values were normally distributed (K-S Z $=0.7-0.8$ ). The ANOVA for the mood ratings yielded a large effect of $\eta^{2}=0.23$ $[\mathrm{F}(3,76)=7.7]$. Whilst in the natural control group and the placebo group mood ratings practically remained the same throughout the experiment $\left(\Delta_{\text {Control }}=1.4\right.$, $\left.\Delta_{\text {Placebo }}=2.2\right)$, the mean differences for both inhaler groups were markedly larger $\left(\Delta_{\text {Balancel }}=6.3\right.$, $\Delta_{\text {Balance2 }}=5.1$ ). Mood ratings in all four groups reflected a scale value that was 'moderate' at baseline, but it increased in both inhaler groups to a value that was 'pretty much' (Table 1). Mean comparisons revealed that both control groups did not differ statistically $(\mathrm{d}=0.2$; CI: $-3.8<\mathrm{d}<0.8)$. However, mean difference between the natural control group and the Balance 1 group was large $(\mathrm{d}=1.2$; CI: $0.5<\mathrm{d}<1.9)$, as was that between the placebo group and the Balance 1 group ( $\mathrm{d}=1.1 ;$ CI: $0.4<\mathrm{d}<1.7$ ). Likewise, the respective difference between the Balance 2 group and the natural control group was $\mathrm{d}=1.0(\mathrm{CI}: 0.4<\mathrm{d}<1.7)$ and $\mathrm{d}=0.9$ (CI: $0.2<\mathrm{d}<1.5$ ) for the comparison with the placebo group. There was no statistical difference between the two inhaler groups $(\mathrm{d}=0.2 ; \mathrm{CI}:-0.9<\mathrm{d}<0.3)$. Thus, in both verum groups inhalation of the odor compound enhanced participants' mood by approx. $10 \%$ after 40 minutes and $13 \%$ after 70 minutes.

Additional analyses. Given the fact that in both control conditions 5-HT levels dropped from baseline to post treatment measurement, the specific effect of the inhaler could have been an overestimation. To account for that, mean comparisons between the baseline and post treatment samples were performed. The analyses revealed that the decrease of 5-HT in the natural control condition was statistically negligible ( $\mathrm{d}=0.1 ; \mathrm{CI}:-0.5<\mathrm{d}<0.8)$. The decrease of $5-\mathrm{HT}$ in the placebo group was statistically medium in size but fell within a positive and negative confidence interval ( $\mathrm{d}=0.4$; CI: $-0.3<\mathrm{d}<1$ ). Hence, there was no indication that the inhalers' effect was overestimated.

Although neurotransmitter levels are best measured in the cerebrospinal fluid, serotonin concentrations in the body and brain are regarded to be highly correlated (Audhya et al. 2012). In this study, the increase of serotonin in the body caused by the inhaler should have co-varied with the increase of serotonin in the brain, especially when considering the fact that scent molecules directly target central neuropsychological pathways. To explore the relationship between neuroendocrine and psychological factors, correlational analyses were performed between the mood ratings and urine 5-HT excretion. The results showed that post intervention mood ratings and post treatment urine serotonin levels yielded a large correlational effect of $\mathrm{r}=0.4(\mathrm{~d}=0.8)$ for the combined verum samples (Balance 1 and 2), whilst this relationship was inverse for individuals in the natural control and placebo group $(r=-0.2$ : $\mathrm{d}=0.4$ ). The difference between these two correlations was large (Cohen's $q=0.6$ ) and supported the notion that the inhaler's effect on mood was likely to have been modulated by an increased serotonin release in the brain. Likewise, further correlational analyses showed that in both inhaler conditions $(n=40)$, post treatment cortisol was negatively correlated with serotonin $(\mathrm{r}=-0.2 ; \mathrm{d}=0.4)$, whilst for the control conditions $(\mathrm{n}=40)$ there was no such relationship $(\mathrm{r}=0.02 ; \mathrm{d}=0.04)$. This was corroborated by a multiple correlation analysis with post treatment mood ratings as the dependent variable and the difference scores for serotonin and cortisol as independent variables that yielded a large effect of $|\mathrm{R}|=0.5 \quad\left(\mathrm{R}^{2}=0.25\right.$; $\left.\eta^{2}=0.25, \mathrm{~F}[2,39]=6.3\right)$.

\section{Discussion}

The pilot study confirmed the assumption that the specially formulated essential oil composition inhaled several times directly into the nose increases the excretion of the urine serotonin after a 70-minute time lapse. This result is noteworthy because, unlike e.g., cardiovascular parameters, which respond very quickly to direct odor inhalations, urine serotonin excretion depends on several factors that may not reflect a linear and/or fast acting relationship due to the complexity of 5-HT catabolization and/or reabsorption in the kidneys. In addition, although there appear to be significant interindividual differences with regard to serotonin reabsorption into the blood and excretion in the urine, which may conceal or bias any specific effect on serotonin release in the body, the relatively large effect in the pilot study would 
indicate a high specificity of the inhaler. However, confounding factors such as responsiveness or placebo effects might have produced the result. To rule out such confounders, the main study was conducted using four experimental arms. It included additional sampling of cortisol, employing a pseudoplacebo condition and a natural control condition to determine the specific role of natural odors, and varying the time of sampling to investigate temporal effects of 5-HT excretion.

The main study confirmed and extended the findings of the pilot study. It showed that the inhaler tested (AromaStick ${ }^{\bullet}$ Balance), which effectively improved neurocardiac mechanisms in previous studies, also affects neuroendocrine mechanisms involving serotonin release. Direct delivery of essential oil molecules to the nose increased urine serotonin efflux by about $28 \%$ during a 70 -minute window involving six inhalation cycles. The size of the differential effects warrants mentioning for three reasons. Firstly, it indicated a highly specific causation that was not affected by natural serotonin fluctuation or non-specific effects like expectation or pleasantness of the scent composition. Secondly, unlike dietary manipulation of 5-HT release, for instance with 5-HTP, which peaks as a function of the size and regularity of the dose (Joy et al. 2008), the inhaler produced a marked spot urine serotonin excretion with very small, but highly concentrated doses of scent molecules. Thirdly, the scent molecules, which most likely targeted serotonin release in the body via nervous and/or humoral pathways, increased urine excretion to a degree that was larger than the commonly assessed $24 \mathrm{~h}$ urine serotonin excretion described in the literature for tryptophan-rich foods and certain medications (Feldman and Lee 1985; Nuttall and Pingree 1998). Despite the large mean effects, the analyses also revealed substantial interindividual differences with regard to response rates. In some individuals, sniffing did not produce significant increases of urine serotonin while in others the increments were remarkable. This effect was larger in the second study and probably due to the number of individuals included in the analysis. Nonetheless, the fact that an increase of approximately $18 \%$ occurred after inhaling three times supported the notion that the olfactory system provides a swift and effective way to release serotonin. It would appear, however, that the effect builds up as a result of repeated inhalation, although marked effects are already detectable after only a few inhalations.

The main study also provided further insight into the cortisol reducing capability of the inhaler. In line with previous findings (Schneider 2016), only a few inhalations sufficed to reduce cortisol levels even in a sample that was not chronically stressed or exposed to distinct experimental stressors. Unlike serotonin release, however, there might be a non-linear relationship between quantity of sniffing and cortisol decrease such that the effect abates after repeated use. In this sample, cortisol levels were not overly high and a reduction of relatively normal levels might be physiologically difficult to sustain. Still, the relationship between urinary serotonin and salivary cortisol indicated that the inhaler targeted two antagonistic endocrine systems which improved individuals' subjective affective state.

This work has a number of potential limitations. Firstly, although the inhaler's use was standardized in terms of the duration of inhalation phases, it could not be standardized with regard to the amount of air inhaled (for instance by a special mask controlling for air molecule concentration). Depending on the user's lung capacity, interindividual differences in the amount of air inhaled with the AromaStick ${ }^{\circ}$ are to be expected. However, the inhaler was tested 'as is', (i.e., as a ready-to-use tool in everyday life) and not as a drug delivering exact pharmaceutical doses per unit. As such, the results of the study address the inhaler's principal potency to affect serotonin release. Secondly, the experimental setting did not involve stressors and thus it is unclear whether similar results could be expected after being exposed to mental or physical stressors. Future studies should explore this issue and specifically test additional factors like e.g., number of inhalations, short-term and long-term effects, and interindividual responsiveness. Thirdly, although the inhaler proved to be superior to the natural control and the placebo treatment, it remains to find out whether these effects translate to the clinical realm. Therefore, it would be useful to compare it in a clinical population with either supplements containing high doses of 5-HTP or active drugs (i.e., SSRIs). Although the effects observed were large in statistical terms and produced practically relevant mood-elevating effects, it should be empirically established if the inhaler can produce similar results in persons with dysfunctional or down-regulated affective states. It should again be noted, however that the inhaler is not a medical product and therefore is not intended to be used in clinical settings.

\section{Conclusions}

Recapitulating the main objectives of this work, the following findings were obtained: (a) the neuro- 
endocrinological effect of the essential oil inhaler AromaStick 'Balance' was confirmed to increase serotonin and decrease cortisol release; (b) these effects were specific and independent from natural fluctuations or psychological factors, and (c) they occurred after three inhalation cycles of three deep in- and exhalations. Thus, intermittently and directly sniffing natural essential oils may have practical implications for improving affective states via exogenous manipulation of mood-relevant neuromodulators like serotonin and cortisol in everyday life.

\section{Acknowledgements}

The study was partially supported by AromaStick Inc., Sargans, Switzerland. The company had no role in the collection, analysis, and interpretation of data, in the writing of the report, and in the decision to submit the article for publication. To ensure that the principal investigator (RECON) was not biased with regard to the outcome, it was made sure that he did not interfere with the actual treatments and had no contact with the participants. I thank Kirsty Scott for helpful comments on the paper's intelligibility.

\section{References}

Agatonovic-Kustrin S, Kustrin E, Gegechkori V, Morton DW. Anxiolytic terpenoids and aromatherapy for anxiety and depression. Adv Exp Med Biol 1260, 283-296, 2020.

Audhya T, Adams JB, Johansen L. Correlation of serotonin levels in CSF, platelets, plasma, and urine. Biochim Biophys Acta 1820, 1496-1501, 2012.

Bellono NW, Bayrer JR, Leitch DB, Castro J, Zhang C, O'Donnell TA, Brierley SM, Ingraham HA, Julius D. Enterochromaffin cells are gut chemosensors that couple to sensory neural pathways. Cell 170, 185-198, 2017.

Berger M, Gray JA, Roth BL. The expanded biology of serotonin. Annu Rev Med 60, 355-366, 2009.

Borenstein M, Hedges LV, Higgins JPT, Rothstein HR. Introduction to meta-analysis. John Wiley \& Sons, Chichester, 2009.

Braun T, Voland P, Kunz L, Prinz C, Gratzl M. Enterochromaffin cells of the human gut: Sensors for spices and odorants. Gastroenterology 132, 1890-1901, 2007.

Cohen J. Statistical power analysis for the behavioral sciences. Laurence Erlbaum Associates, Hillsdale, 2008.

Del Colle A, Israelyan N, Gross Margolis K. Novel aspects of enteric serotonergic signaling in health and brain-gut disease. Am J Physiol Gastrointest Liver Physiol 318, G130-G143, 2020.

Edris AE. Pharmaceutical and therapeutic potentials of essential oils and their individual volatile constituents: A review. Phytother Res 21, 308-323, 2007.

Faul F, Erdfelder E, Buchner A, Lang A-G. Statistical power analyses using G*Power 3.1: Tests for correlation and regression analyses. Behav Res Methods 41, 1149-1160, 2009.

Feldman JM, Lee EM. Serotonin content of foods: effect on urinary excretion of 5-hydroxyindoleacetic acid. Am J Clin Nutr 42, 639-643, 1985.

Frazer A, Hensler JG. Understanding the neuroanatomical organization of serotonergic cells in the brain provides insight into the functions of this neurotransmitter. In: Basic Neurochemistry (Eds. GJ Siegel, BW Agranoff, RW Albers, SL Fisher, MD Uhler), pp. 264-268. Lippincott-Raven, Philadelphia, 1999.

Greenland S, Senn SJ, Rothmann KJ, Carlin JB, Poole C, Goodman SN, Altman D.G. Statistical tests, P values, confidence intervals, and power: a guide to misinterpretations. Eur J Epidemiol 31, 337-350, 2016.

Hinz M, Stein A, Uncini T. 5-HTP efficacy and contraindications. Neuropsychiatr Dis Treat 8, 323-328, 2012.

Hunter, JE, Schmidt F. Methods of Meta-Analysis: Correcting error and bias in research findings. Sage Publishers, London, 2004.

Hur MH, Song JA, Lee J, Lee, MS. Aromatherapy for stress reduction in healthy adults: A systematic review and meta-analysis of randomized clinical trials. Maturitas 79, 362-369, 2014.

Hwang S, Shin, S. The effects of aromatherapy on sleep improvement: A systematic literature review and meta-analysis. J Altern Complement Med 21, 61-68, 2015.

Joy T, Walsh G, Tokmakejian S, Van Uum SH. Increase of urinary 5-hydroxyindoleacetic acid excretion but not serum chromogranin A following over-the-counter 5-hydroxytryptophan intake. Can J Gastroenterol 22, $49-53,2008$.

Kiecolt-Glaser JK, Graham JE, Malarkey WB, Porter K, Lemeshow S, Glaser R. Olfactory influences on mood and autonomic, endocrine, and immune function. Psychoneuroendocrinology 33, 328-339, 2009.

Konturek PC, Konturek SJ, Ochmanski W. Neuroendocrinology of gastric H+ and duodenal HCO3- secretion: the role of brain-gut axis. Eur J Pharmacol 499, 15-27, 2004. 
Locher C, Koechlin H, Zion SR, Werner C, Pine DS, Kirsch, I, Kessler RC, Kossowsky J. Efficacy and safety of Selective Serotonin Reuptake Inhibitors, Serotonin-Norepinephrine Reuptake Inhibitors, and placebo for common psychiatric disorders among children and adolescents. JAMA Psychiatry 74, 1011-1020, 2017.

Lv XN, Liu ZJ, Zhang HJ, Tzeng CM. Aromatherapy and the central nerve system (CNS): therapeutic mechanism and its associated genes. Curr Drug Targets 14, 872-879, 2013.

Mahar I, Bambico FR, Mechawar N, Nobrega JN. Stress, serotonin, and hippocampal neurogenesis in relation to depression and antidepressant effects. Neurosci Biobehav Rev 38, 173-192, 2014.

Masaoka Y, Homma I. Breathing for olfaction and emotion. In: The biology of odors, sources, olfaction and response (Eds. LE Weiss, M Atwood), pp. 295-307, Novapublishers, New York, 2011.

Nakumara A, Fujiwara S, Matsumoto I, Abe K. tress repression in restrained rats by (R)-(-)-linalool inhalation and gene expression profiling of their whole blood cells. J Agric Food Chem 57, 5480-5485, 2009.

Nuttall KL, Pingree SS. The incidence of elevations in urine 5-hydroxyindoleacetic acid. Ann Clin Lab Sci 28, 167$174,1998$.

Sanchez-Vidana DI, Pui-Ching NS, He W, Chow JKW, Lau BWM, Tsang HWH. The effectiveness of aromatherapy for depressive symptoms: A systematic review. Evid Based Complement Alternat Med 2017, 5869315, 2017.

Santarsieri D, Schwartz T L. Antidepressant efficacy and side-effect burden: A quick guide for clinicians. Drugs Context 4, 1-12, 2015.

Schneider R. There is something in the air: Testing the efficacy of a new olfactory stress relief method (AromaStick ${ }^{\circledR}$. Stress \& Health 32, 411-426, 2016.

Schneider R. A breath of fresh air: Arterial blood oxygen saturation is increased upon the use of an essential oil inhaler (AromaStick ${ }^{\circledR}$ ). Results from a prospective, controlled, experimental study involving healthy individuals. Curr Respir Med Rev 13, 213-220, 2017.

Schneider R, Singer N, Singer T. Medical aromatherapy revisited - Basic mechanisms, critique, and a new development. Hum Psychopharmacol Clin Exp 34, e2683, 2019.

Singer J. Composition for use in an inhalation device and inhalation device useful thereof. US Patent US2016/0279358 A1, 2016.

Strasser B, Gostner JM, Fuchs D. Mood, food, and cognition: role of tryptophan and serotonin. Curr Opin Clin Nutr Metab Care 19, 55-61, 2016.

Tafet GE, Idoyaga-Vargas VP, Abulafia DP, Calandria JM, Roffman SS, Chiovetta A, Shinitzky M. Correlation between cortisol level and serotonin uptake in patients with chronic stress and depression. Cogn Affect Behav Neurosci 1, 388-393, 2001.

Trachte GJ, Uncine T, Hinz M. Both stimulatory and inhibitory effects of dietary 5-hydroxytryptophan and tyrosine are found on urinary excretion of serotonin and dopamine in a large human population. Neuropsychiatr Dis Treat 5, 227-235, 2009.

Yabut JM, Crane JD, Green AE, Keating DJ, Khan WI, Steinberg GR. Emerging roles for serotonin in regulating metabolism: new implications for an ancient molecule. Endocrine Rev 40, 1092-1107, 2019.

Zhang N, Zhang L, Feng L, Yao L. Cananga odorata essential oil reverses the anxiety induced by 1-(3-chlorophenyl) piperazine through regulating the MAPK pathway and serotonin system in mice. J Ethnopharmacol 219, 23-30, 2018 